\title{
PITX1 is a novel predictor of the response to chemotherapy in head and neck squamous cell carcinoma
}

\author{
MASAO TAKENOBU ${ }^{1,2}$, MITSUHIKO OSAKI ${ }^{2,3}$, KAZUNORI FUJIWARA ${ }^{1}$, TAKAHIRO FUKUHARA ${ }^{1}$, \\ HIROYA KITANO $^{1}$, HIROYUKI KUGOH ${ }^{3,4}$ and FUTOSHI OKADA ${ }^{2,3}$ \\ ${ }^{1}$ Department of Otolaryngology, Head and Neck Surgery; ${ }^{2}$ Division of Pathological Biochemistry, \\ Department of Biomedical Sciences, Faculty of Medicine; ${ }^{3}$ Chromosome Engineering Research Center, \\ ${ }^{4}$ Division of Molecular Genetics and Biofunction, Graduate School of Medical Science; \\ Tottori University, Yonago, Tottori 683-8503, Japan
}

Received August 28, 2015; Accepted February 19, 2016

DOI: $10.3892 / \mathrm{mco} .2016 .880$

\begin{abstract}
The pituitary homeobox 1 (PITX1) protein is essential for developmental processes in humans. Previously, PITX1 was identified as a possible tumor suppressor gene in various types of human carcinoma. However, the association between PITX1 and human head and neck squamous cell carcinoma (HNSCC) remains to be elucidated. Immunohistochemical analysis was performed to examine the expression levels of PITX1 in 47 cases of HNSCC, and in 4 control cases. The expression of p53 was also examined in these cases. The labeling indices (LIs) were calculated, and the correlations between clinical factors (chemosensitivity, prognosis and the degree of differentiation) and the LIs were assessed. The PITX1 LI in HNSCC was $27.4 \pm 14.5 \%$, which was significantly lower compared with the LIs of the control samples: $76.9 \pm 6.97 \%(\mathrm{P}<0.05)$. Additionally, the PITX1 LIs were $39.9 \pm 6.2,26.9 \pm 16.9$ and $24.2 \pm 11.8 \%$ in the complete response $(\mathrm{CR})$, partial response (PR), stable disease or progressive disease (SD/PD) groups, respectively. The PITX1 $\mathrm{LI}$ in the CR group revealed the highest result between the all groups, and it was significantly greater compared with that in the $\mathrm{SD} / \mathrm{PD}$ group $(\mathrm{P}<0.01)$. The $\mathrm{p} 53$ LIs were 24.5 \pm 19.9 , $25.7 \pm 16.9$ and $19.8 \pm 13.8$ in the $\mathrm{CR}, \mathrm{PR}$ and $\mathrm{SD} / \mathrm{PD}$ groups, respectively $(\mathrm{P}>0.05)$. Neither the PITX1 nor the p53 LIs were a statistically significant indicator of the prognosis. PITX1 is a candidate tumor suppressor gene and a possible predictive biomarker of chemosensitivity of human HNSCC.
\end{abstract}

Correspondence to: Dr Mitsuhiko Osaki, Division of Pathological Biochemistry, Department of Biomedical Sciences, Faculty of Medicine, Tottori University, 86 Nishi-Cho, Yonago, Tottori 683-8503, Japan

E-mail: osamitsu@med.tottori-u.ac.jp

Key words: PITX1, head and neck squamous cell carcinoma, tumor suppressor gene, biomarker, chemosensitivity

\section{Introduction}

Head and neck squamous cell carcinoma (HNSCC) is a devastating disease and the sixth most common cancer type worldwide, responsible for $>500,000$ new cases annually. Despite progression in diagnostic and therapeutic modalities, $\sim 60 \%$ of patients present with locally advanced disease, and the five-year survival rate is $\sim 50 \%$ (1-3). The main strategies of therapy are chemoradiation therapy or surgery. Neoadjuvant or induction chemotherapy, followed by local therapy, is often performed and it was reported that the response to induction chemotherapy may be a factor that can predict the efficacy of chemoradiation therapy (4). However, no clinically validated biomarkers of HNSCC exist for predicting prognosis or treatment outcome. This may be one of the reasons for the low survival rate or the occurrences of unsuccessful treatment. Therefore, it is expected that the identification of novel biomarkers of HNSCC will assist with determining prognosis more accurately or increase the efficacy of treatment, which may provide the patients with more options.

The pituitary homeobox 1 (PITX1) protein was originally described as a member of the bicoid-associated homeobox transcription factor family that is involved in the transcriptional regulation of proopiomelanocortin gene in the adult pituitary gland (5). PITX1 is important in the development of hind limbs and determines morphological features of the muscles, tendons and bones of the hind limbs (6). In addition, PITX1 has been identified as a suppressor of RAS activity and tumorigenicity (7). Previously, it was reported that PITX1 is a negative regulator of telomerase, due to transcriptional repression of the promoter of telomerase reverse transcriptase (TERT) (8). Downregulation of PITX1 is consistently associated with the malignancy of various human cancer types, including oral squamous cell carcinoma (9), malignant melanoma (10), esophageal (11), gastric $(8,12)$, lung (13), breast (14), hepatic (15), colorectal (16), and prostate (17) cancer, suggesting that PITX1 may be a tumor suppressor gene. By contrast, an association between PITX1 and HNSCC, including pharyngeal or laryngeal carcinoma, remains to be identified.

In the present study, the expression of PITX1 and its clinical significance in HNSCC was investigated. The present 
study also examined the association of a frequently reported, but not validated in HNSCC, p53 gene mutation (18-24). The clinical utility of these biomarkers was then determined in HNSCC.

\section{Materials and methods}

Tissue samples. Tissue samples from biopsies of $47 \mathrm{HNSCCs}$ were collected prior to the administration of any treatment. All cases were diagnosed as HNSCC for the first time at the Department of Otolaryngology, Head and Neck Surgery, Faculty of Medicine, Tottori University (Yonago, Japan) between January 2008 and December 2012. The cases included 21 laryngeal carcinomas, 16 hypopharyngeal carcinomas, 5 oropharyngeal carcinomas and 5 oral carcinomas. Of these 47 cases, 41 were at stage III or IV, and 6 were at stage I or II. All the patients received induction chemotherapy comprising cisplatin, 5-fluorouracil and docetaxel, followed by a local therapy. In the case of patients $>65$-years-old, nedaplatin was used instead of cisplatin. A total of 4 normal samples of oropharyngeal tissue served as controls. Approval for the present study was obtained from the Institutional Review Board of the Faculty of Medicine, Tottori University (Approval No. 2334). All specimens were fixed in 10\% formalin (Wako Pure Chemical Industries, Ltd., Osaka, Japan) and embedded in paraffin (Wako Pure ChemicalIndustries, Ltd.). Subsequently, $4 \mu \mathrm{m}$-thick sections were prepared with a microtome and examined using immunohistochemistry.

Immunohistochemistry. Dewaxed paraffin-embedded sections were immunostained using the streptavidin-biotin peroxidase complex method with a HISTOFINE SAB-PO Immunohistochemical Staining kit (Nichirei Biosciences, Inc., Tokyo, Japan). The sections were immersed in $3 \%$ hydrogen peroxide in phosphate-buffered saline for $60 \mathrm{~min}$ to bleach the melanin dye and block endogenous peroxidase activity. Antigen retrieval was performed by autoclaving with $10 \mathrm{mM}$ citrate buffer $(\mathrm{pH} \mathrm{6.0)}$ for $10 \mathrm{~min}$. The primary antibodies used were as follows: Rabbit polyclonal anti-PITX1 (1:500, Abcam, Cambridge, UK) and mouse monoclonal anti-p53 (DO7; 1:50; Dako, Glostrup, Denmark), which is thought to detect the p53 gene mutation. Immunoreactions were visualized with diaminobenzidine (DakoCytomation, Glostrup, Denmark) and the sections were counterstained with hematoxylin (Wako Pure Chemical Industries, Ltd.). Evaluation of the immunoreactive cells was performed using a light microscope (Eclipse E400; Nikon, Tokyo, Japan; magnification, $\mathrm{x} 400$ ) in four random visual fields in each slide. In the HNSCC cases, the percentage of immunoreactive cells among the tumor cells was calculated, and in the control cases, the percentage of those among normal squamous cells was calculated. The percentage of immunoreactive cells was defined as the labeling index (LI) for each primary antibody.

Evaluation of the response to chemotherapy. Following one cycle of neoadjuvant chemotherapy, computed tomography or magnetic resonance imaging was performed. The response to chemotherapy was subsequently determined, according
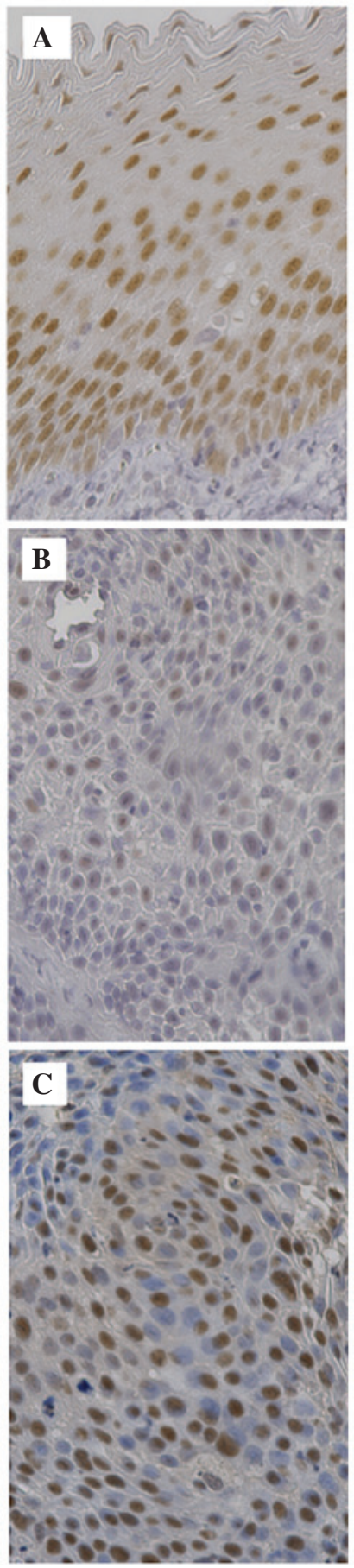

Figure 1.Immunohistochemicalexamination of PITX1.Immunohistochemical analysis of the expression of PITX1 was performed in (A) normal oropharyngeal mucosa and (B) HNSCC. (C) Immunohistochemical examination of the expression of p53 in the HNSCC; magnification, $\mathrm{x} 400$. PITX1, pituitary homeobox 1; HNSCC, head and neck squamous cell carcinoma.

to the RECIST guidelines (25) and classified as a complete response (CR), partial response (PR), or stable disease (SD) or progressive disease $(\mathrm{PD})$.

Statistical analysis. The Mann-Whitney U test was used to compare the expression of PITX1 in HNSCC with that of the control cases and to evaluate the association between the expression of PITX1 or p53, and clinical factors, including the response to chemotherapy, prognosis and degree of 


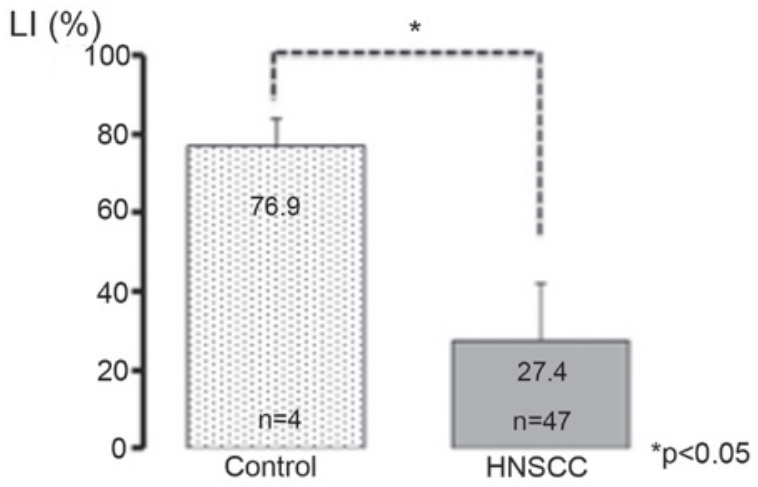

Figure 2. Expression of PITX1 in HNSCC. The expression of PITX1 was significantly lower in the HNSCC lesions compared with in normal mucosae. The data are presented as the mean \pm standard deviation $(\mathrm{P}<0.05)$. PITX1, pituitary homeobox 1; HNSCC, head and neck squamous cell carcinoma.

A

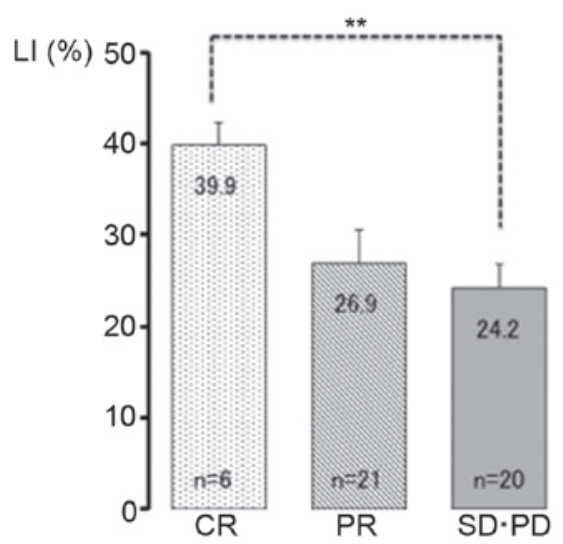

B

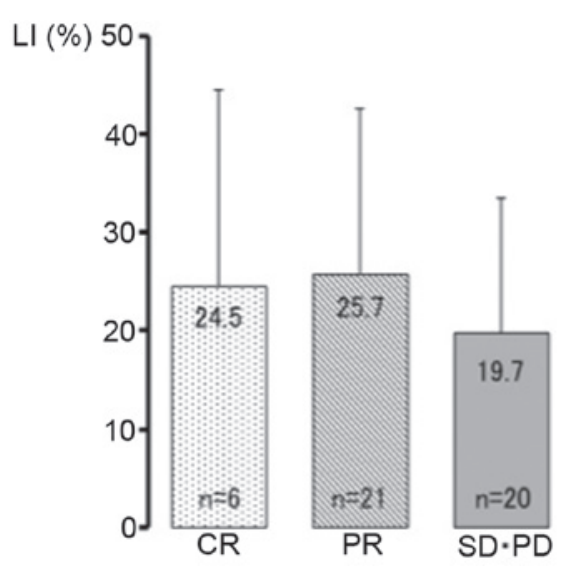

Figure 3. Correlation between the expression of PITX1 or p53, and the response to chemotherapy. (A) The PITX1 LI in tumor specimens corresponding to a $\mathrm{CR}$ was significantly higher compared with that in $\mathrm{SD}$ or $\mathrm{PD}$. The data are presented as the mean \pm standard deviation $\left({ }^{* *} \mathrm{P}<0.01\right)$. (B) No significant difference in the p53 LI were observed between the three groups. The data are presented as the mean \pm standard deviation. LI, labeling indices; PITX1, pituitary homeobox 1; CR, complete response; PR, partial response; $\mathrm{SD}$, stable disease; $\mathrm{PD}$, progressive disease.

differentiation. Kaplan-Meier analysis was also used to assess the overall survival and disease-free survival. The differences in survival rates were assessed by means of the log-rank test. The data are presented as the mean \pm standard

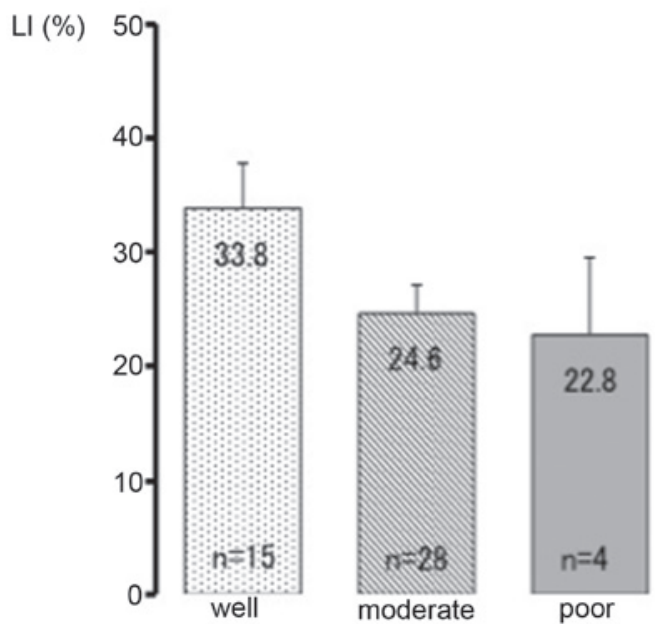

Figure 4. Correlation between the expression of PITX1 and the degree of differentiation. No significant difference was observed between the well, moderate and poor differentiated groups. The data are presented as the mean \pm standard deviation. LI, labeling indices; PITX1, pituitary homeobox 1 .

deviation. $\mathrm{P}<0.05$ was considered to indicate a statistically significant difference.

\section{Results}

Expression levels of PITX1 and p53 in HNSCC. Immunohistochemical analysis revealed that the expression of PITX1 in the samples of normal pharyngeal tissue was clearly visible as a brown nucleus in the squamous-epithelium cells, particularly in the basal cell layer (Fig. 1A). By contrast, PITX1 expression in the HNSCC samples was detected less in a smaller percentage of cells compared with in the normal control (Fig. 1B). The PITX1 LI was $76.9 \pm 6.97 \%$ in the control pharyngeal samples and $27.4 \pm 14.5 \%$ in the HNSCC samples (Fig. 2). This difference was statistically significant $(\mathrm{P}<0.05)$.

Immunohistochemical analysis also revealed that p53 expression was clearly observed in the nuclei of HNSCC cells (Fig. 1C), however, not in normal pharyngeal epithelial cells. The p53 LI was $23.0 \pm 15.4 \%$ in the HNSCC samples.

Correlation between the expression levels of PITX1 or p53 and the response to chemotherapy. Of the 47 HNSCC patients, 6 patients exhibited a CR, 21 exhibited a PR, 19 exhibited $\mathrm{SD}$ and 1 exhibited a PD. The PITX1 LIs were 39.9 \pm 6.2 , $26.9 \pm 16.9$ and $24.2 \pm 11.8 \%$ in the CR, PR and SD/PD groups, respectively (Fig. 3A). The PITX1 LI in the CR group exhibited the highest result among all groups and was significantly higher compared with that of the SD/PD groups $(\mathrm{P}<0.01)$. The p53 LIs were $24.5 \pm 19.9,25.7 \pm 16.9$ and $19.8 \pm 13.8 \%$ in the CR, $\mathrm{PR}$ and SD/PD groups, respectively (Fig. 3B). No significant differences were observed between the groups.

Correlation between the expression of PITX1 and the degree of differentiation. Of the 47 cases, 15 exhibited well differentiated carcinoma, 28 exhibited moderately differentiated carcinoma and 4 exhibited poorly differentiated carcinoma. The PITX1 LIs were 33.8 $\pm 15.2,24.6 \pm 13.6$ and $22.8 \pm 13.4 \%$ in the well differentiated, moderately differentiated, and poorly 


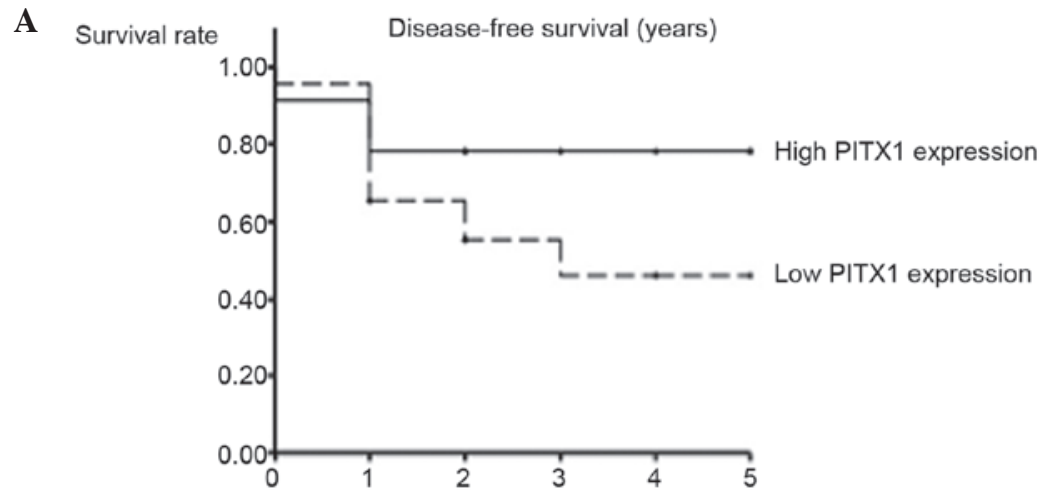

B

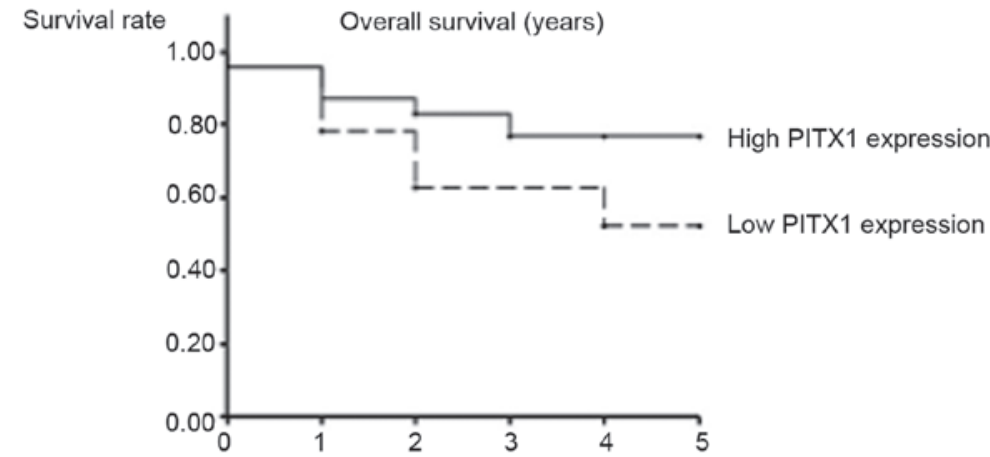

C

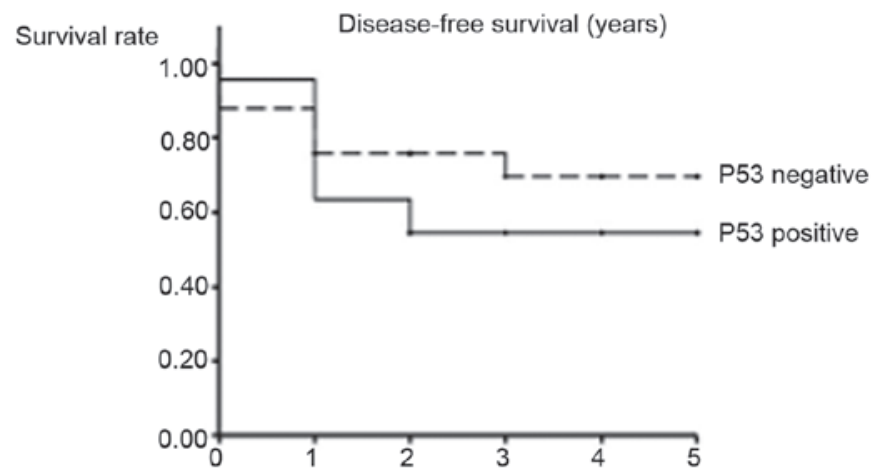

D

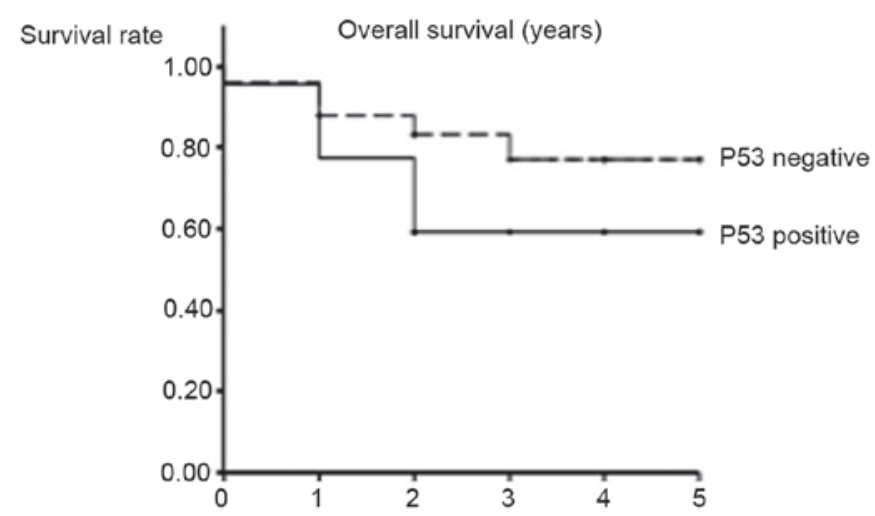

Figure 5. Correlation between the expression of PITX1 and (A) disease-free survival and (B) overall survival. Correlation between the p53 mutation and (C) disease-free survival and (D) overall survival. Although a higher expression of PITX1 or the absence of the p53 mutation was associated with an improved prognosis, no statistically significant differences were observed in the analyses (A) $\chi^{2}=3.06, P=0.08 ;(B) \chi^{2}=2.20, P=0.14,(C) \chi^{2}=1.08, P=0.30$ and $(\mathrm{D}) \chi^{2}=2.07$, $\mathrm{P}=0.15)$. PITX1, pituitary homeobox 1 . 
differentiated groups, respectively (Fig. 4). No significant differences were observed between these subgroups.

Correlation between the expression of either PITX1 or p53 and prognosis. To compare the differences in disease-free survival and overall survival, the HNSCC samples were separated into two groups by PITX1 LIs. The samples whose LIs were $<28.9$ (the median LI of all HNSCC samples) were designated as the low PITX1 expression group, and the other samples were designated as the high PITX1 expression group. Although the higher expression of PITX1 tended to be associated with an improved prognosis, no significant differences were observed between the two groups $\left(\chi^{2}=3.06\right.$ and $\mathrm{P}=0.08$ for disease-free survival and $\chi^{2}=2.20$ and $\mathrm{P}=0.14$ for overall survival; Fig. 5A and B). Additionally, the samples whose LI was $<20.0$ were designated as the $p 53$ mutation negative $(\mathrm{n}=25)$ group, and the others termed the $p 53$ mutation positive $(\mathrm{n}=22)$ group. No significant differences were observed between these two groups with regards to disease-free survival $\left(\chi^{2}=1.08 ; \mathrm{P}=0.30\right)$ overall survival ( $\chi^{2}=2.07 ; \mathrm{P}=0.15$; Fig. $5 \mathrm{C}$ and $\left.\mathrm{D}\right)$.

\section{Discussion}

The present study demonstrated that the expression of PITX1 was downregulated in HNSCC samples compared with that in normal pharyngeal samples, suggesting that PITX1 may act as a tumor suppressor and a possible novel biomarker in HNSCC and other human malignancies (8-17). A previous study demonstrated that a low expression of PITX1 is associated with a poor prognosis in colorectal carcinoma (16) and hepatocellular carcinoma (15). It was reported that a low expression of PITX1 is also associated with poor differentiation of gastric cancer (12) and oral squamous cell carcinoma (9). However, the clinical significance of PITX1 in HNSCC remains unclear. Therefore, the present study assessed this significance and the potential use of PITX1 as a novel biomarker of HNSCC.

The present results revealed that the expression of PITX1 was significantly higher in the CR group compared with the SD/PD group, suggesting that higher expression of PITX1 is associated with higher chemosensitivity of HNSCC. It is known that histological differentiation is associated with the chemosensitivity of HNSCC (26) and that the level of PITX1 expression correlates with differentiation of oral squamous cell carcinoma (9) and human gastric cancer (12). Accordingly, the correlation between the expression of PITX1 and the degree of differentiation was assessed. No statistically significant result was obtained. These data markedly suggested that PITX1 is a possible biomarker for predicting chemosensitivity, independently from histological differentiation of HNSCC.

It is known that $p 53$ is a commonly mutated tumor suppressor gene in HNSCC (18). Several previous studies have shown that a p53 gene mutation is a prognostic biomarker of HNSCC (19-21) and that a $p 53$ mutation is associated with the risk of a poor response to chemotherapy $(22,23)$. By contrast, it was also reported that a $p 53$ mutation is not significantly associated with prognosis (24) or chemosensitivity (27) in HNSCC. According to the present results, neither PITX1 nor p53 are statistically significant predictors of overall survival and disease-free survival. The $p 53$ mutation is not significantly associated with chemosensitivity in the present study, although the differences from the previous reports in the design of chemotherapy must be taken into consideration. Therefore, the expression of $\mathrm{p} 53$ as a clinical biomarker in HNSCC remains controversial.

The present study is the first, to the best of our knowledge, to describe the expression of PITX1 and a correlation between the expression of PITX1 and the chemosensitivity of HNSCC. However, the mechanisms that underlie the association between PITX1 and chemosensitivity remain unclear. Previously, PITX1 was identified as a tumor suppressor that downregulates the RAS pathway by acting on RASAL1, a member of the RAS-GTPase activating protein family (7). The protein expression of wild-type KRAS2 is a major determinant of the proliferation of HNSCC cells. Amplification of unmutated KRAS2 in HNSCC contributes to tumor growth (28). It was also reported that PITX1 suppresses TERT by directly binding to the TERT promoter (8), which is known to be a component of the telomerase enzyme and is necessary for cancer cell immortalization and proliferation (29). Previously, Ohira et al (30) reported that the mRNA expression level of PITX1 is directly suppressed by microRNA-19b, followed by the upregulation of TERT expression in melanoma cells (30). In addition, certain previous reports have shown that upregulated telomerase activation, as well as the mRNA expression of TERT, correlates with poor chemosensitivity (31-33). These previous reports appear to support the hypothesis that attenuating the expression of PITX1 by miR-19b may be associated with malignancy and may assist with chemoresistance via telomerase activation in HNSCC cells.

The present study is the first, to the best of our knowledge, to report an association between PITX1 and HNSCC. The downregulation of PITX1 in head and neck squamous epithelial cells may be involved in the carcinogenesis of HNSCC. Therefore, PITX1 is considered a candidate tumor suppressor gene. Additionally, PITX1 may serve as a novel biomarker for predicting the response to chemotherapy in HNSCC. Future investigaitons are required to determine the potential role of PITX1 for modulating chemosensitivity in HNSCC cells.

\section{Acknowledgements}

The present study was supported by JSPS KAKENHI (no. 22501012).

\section{References}

1. Jemal A, Siegel R, Ward E, Hao Y, Xu J and Thun MJ: Cancer statistics, 2009. CA Cancer J Clin 59: 225-249, 2009.

2. Al-Sarraf M: Treatment of locally advanced head and neck cancer: Historical and critical review. Cancer Control 9: 387-399, 2002.

3. Seiwert TY and Cohen EEW: State-of-the-art management of locally advanced head and neck cancer. Br J Cancer 92: 1341-1348, 2005.

4. Urba S, Wolf G, Eisbruch A, Worden F, Lee J, Bradford C, Teknos T, Chepeha D, Prince M, Hogikyan N and Taylor J: Single-cycle induction chemotherapy selects patients with advanced laryngeal cancer for combined chemoradiation: A new treatment paradigm. J Clin Oncol 24: 593-598, 2006.

5. Lamonerie T, Tremblay JJ, Lanctôt C, Therrien M, Gauthier Y and Drouin J: Ptx1, a bicoid-related homeo box transcription factor involved in transcription of the pro-opiomelanocortin gene. Genes Dev 10: 1284-1295, 1996. 
6. DeLaurier A, Schweitzer R and Logan M: Pitx1 determines the morphology of muscle, tendon and bones of the hindlimb. Dev Biol 299: 22-34, 2006.

7. Kolfschoten IGM, van Leeuwen B, Berns K, Mullenders J, Beijersbergen RL, Bernards R, Voorhoeve PM and Agami R: A genetic screen identifies PITX1 as a suppressor of RAS activity and tumorigenicity. Cell 121: 849-858, 2005.

8. Qi DL, Ohhira T, Fujisaki C, Inoue T, Ohta T, Osaki M, Ohshiro E, Seko T, Aoki S, Oshimura M and Kugoh H: Identification of PITX1 as a TERT suppressor gene located on human chromosome 5. Mol Cell Biol 31: 1624-1636, 2011.

9. Libório TN, Acquafreda T, Matizonkas-Antonio LF, Silva-Valenzuela MG, Ferraz AR and Nunes FD: In situ hybridization detection of homeobox genes reveals distinct expression patterns in oral squamous cell carcinomas. Histopathology 58: 225-233, 2011.

10. Osaki M, Chinen H, Yoshida Y, Ohhira T, Sunamura N, Yamamoto O, Ito H, Oshimura M and Kugoh H: Decreased PITX1 gene expression in human cutaneous malignant melanoma and its clinicopathological significance. Eur J Dermatol 23: 344-349, 2013.

11. Lord RV, Brabender J, Wickramasinghe K, DeMeester SR, Holscher A, Schneider PM, Danenberg PV and DeMeester TR: Increased CDX2 and decreased PITX1 homeobox gene expression in Barrett's esophagus and Barrett's-associated adenocarcinoma. Surgery 138: 924-931, 2005

12. Chen YN, Chen H, Xu Y, Zhang X and Luo Y: Expression of pituitary homeobox 1 gene in human gastric carcinogenesis and its clinicopathological significance. World J Gastroenterol 14: 292-297, 2008.

13. Chen Y, Knösel T, Ye F, Pacyna-Gengelbach M, Deutschmann N and Petersen I: Decreased PITX1 homeobox gene expression in human lung cancer. Lung Cancer 55: 287-294, 2007.

14. Stender JD, Stossi F, Funk CC, Charn TH, Barnett DH and Katzenellenbogen BS: The estrogen-regulated transcription factor PITX1 coordinates gene-specific regulation by estrogen receptor-alpha in breast cancer cells. Mol Endocrinol 25 1699-1709, 2011

15. Calvisi DF, Ladu S, Conner EA, Seo D, Hsieh JT, Factor VM and Thorgeirsson SS: Inactivation of Ras GTPase-activating proteins promotes unrestrained activity of wild-type Ras in human liver cancer. J Hepatol 54: 311-319, 2011.

16. Knösel T, Chen Y, Hotovy S, Settmacher U, Altendorf-Hofmann A and Petersen I: Loss of desmocollin 1-3 and homeobox genes PITX1 and CDX2 are associated with tumor progression and survival in colorectal carcinoma. Int J Colorectal Dis 27: 1391-1399, 2012

17. Kwok SC,Liu XM, Mangel P and Daskall: PTX1 (ERGIC2)-VP22 fusion protein upregulates interferon-beta in prostate cancer cell line PC-3. DNA Cell Biol 25: 523-529, 2006.

18. Somers KD, Merrick MA, Lopez ME, Incognito LS, Schechter GL and Casey G: Frequent p53 mutations in head and neck cancer. Cancer Res 52: 5997-6000, 1992.

19. Poeta M, Manola J, Goldwasser MA, Forastiere A, Benoit N, Califano JA, Ridge JA, Goodwin J, Kenady D, Saunders J, et al: TP53 mutations and survival in squamous-cell carcinoma of the head and neck. N Engl J Med 357: 2552-2561, 2007.
20. Warnakulasuriya S, Jia C, Johnson N and Houghton J: p53 and P-glycoprotein expression are significant prognostic markers in advanced head and neck cancer treated with chemo/radiotherapy. J Pathol 191: 33-38, 2000

21. Mannarini L, Bertino G, Morbini P, Villa C and Benazzo M Markers of chemoradiation resistance in patients with locally advanced head and neck squamous cell carcinoma, treated by intra-arterial carboplatin and concurrent radiation. Acta Otorhinolaryngol Ital 27: 173-180, 2007.

22. Temam S, Flahault A, Périé S, Monceaux G, Coulet F, Callard P, Bernaudin JF, St Guily JL and Fouret P: P53 gene status as a predictor of tumor response to induction chemotherapy of patients with locoregionally advanced squamous cell carcinomas of the head and neck. J Clin Oncol 18: 385-394, 2000

23. Okumura K, Hasegawa Y, Harada H, Ishizaki K and Murakami S: A comparative study of p53 status and clinical response to chemotherapy in head and neck cancer. Nagoya Med J 46: 171-180, 2003.

24. Szentkúti G, Dános K, Brauswetter D, Kiszner G, Krenács T, Csákó L, Répássy G and Tamás L: Correlations between prognosis and regional biomarker profiles in head and neck squamous cell carcinomas. Pathol Oncol Res 21: 643-650, 2015.

25. Therasse P, Arbuck SG, Eisenhauer EA, Wanders J, Kaplan RS, Rubinstein L, Verweji J, Van Glabbeke M, van Oosterom AT, Christian MC, Gwyther SG: New guldelines to evaluate the response to treatment in solod tumors. J Natl Cancer Inst 92: 205-216, 2000

26. Nakashima T, Maehara Y, Kohnoe S, Hayashi I and Katsuta Y: Histologic differentiation and chemosensitivity of human head and neck squamous cell carcinomas. Head Neck 12: 406-410, 1990.

27. Hoffmann TK, Sonkoly E, Hauser U, van Lierop A, Whiteside TL, Klussmann JP, Hafner D, Schuler P, Friebe-Hoffmann U, Scheckenbach K, et al: Alterations in the p53 pathway and their association with radio- and chemosensitivity in head and neck squamous cell carcinoma. Oral Oncol 44: 1100-1109, 2008.

28. Hoa M, Davis SL, Ames SJ and Spanjaard RA: Amplification of wild-type $\mathrm{K}$-ras promotes growth of head and neck squamous. Cancer Res 62: 7154-7156, 2002.

29. Blagoev KB: Cell proliferation in the presence of telomerase. PLoS One 4: e4622, 2009.

30. Ohira T, Naohiro S, Nakayama Y, Osaki M, Okada F, Oshimura M and Kugoh H: miR-19b regulates hTERT mRNA expression through targeting PITX1 mRNA in melanoma cells. Sci Rep 5: $8201,2015$.

31. Ueda Y, Hiyama E, Kamimatsuse A, Kamei N, Ogura K and Sueda T: Wnt signaling and telomerase activation of hepatoblastoma: Correlation with chemosensitivity and surgical resectability. J Pediatr Surg 46: 2221-2227, 2011.

32. Wang L, Li PF, Geng M, Cao YC and Yin YC: Correlation between chemosensitivity to anticancer drugs and telomerase reverse transcriptase mRNA expression in gastric cancer. Diagn Pathol 8: 33, 2013

33. Guo X, Wang W, Zhou F, Lu Z, Fang R, Jia F, Bu X, Li R, Zhang B, Wu M and Wei L: siRNA-mediated inhibition of hTERT enhances chemosensitivity of hepatocellular carcinoma. Cancer Biol Ther 7: 1555-1560, 2008. 